MARIM, B.G.; SILVA, D.J.H.; GUIMARÃES, M.A.; BELFORT, G. Sistemas de tutoramento e condução do tomateiro visando produção de frutos para consumo in natura. Horticultura Brasileira, Brasília, v.23, n.4, p.951-955, out-dez 2005.

\title{
Sistemas de tutoramento e condução do tomateiro visando produção de frutos para consumo in natura
}

\author{
${ }^{1}$ Bruno G. Marim; ${ }^{2}$ Derly José H. da Silva; ${ }^{2}$ Marcelo de A. Guimarães; ${ }^{3}$ Gabriel Belfort \\ ${ }^{1}$ UFV, Genética e Melhoramento, 36571-000 Viçosa-MG; ${ }^{2}$ UFV, Depto. Fitotecnia; ${ }^{3}$ UFLA, Genética e Melhoramento, 37200-000 \\ Lavras-MG; E-mail: bgmarim@yahoo.com.br
}

\section{RESUMO}

Os sistemas de tutoramento e condução influem no desenvolvimento da planta do tomateiro e na qualidade do fruto produzido. Para avaliar a influência de três sistemas de tutoramento e dois de condução da planta do tomateiro na produção classificada de frutos comercializáveis, foram conduzidos dois experimentos, de agosto a dezembro/1999 (exp. 1) e de 2000 (exp. 2) em Viçosa (MG). O delineamento foi em blocos ao acaso com três repetições, no esquema fatorial $3 \times 2$, tutoramento e condução, respectivamente. Avaliaramse os seguintes métodos de tutoramento: $\mathrm{T} 1$, tradicional ( $\mathrm{V}$ invertido), T2, triangular e T3, vertical e duas formas de condução, com uma e duas hastes por planta. Independentemente do tratamento, as plantas foram podadas acima do sexto cacho. Observaram-se diferenças entre os sistemas de tutoramento, condução e entre os anos de cultivo em relação às características avaliadas, sendo que ocorreu interação entre estes efeitos apenas para as características produção de frutos comercializáveis e produção total. O tutoramento vertical proporcionou aumento na produção de frutos de tamanho grande e diminuição na produção de frutos de tamanho médio e frutos não comercializáveis, quando comparado com os outros métodos de tutoramento. Independentemente do sistema de tutoramento, o tomateiro cultivado com uma haste produziu mais frutos de tamanho grande, de maior valor comercial, enquanto as plantas conduzidas com duas hastes produziram mais frutos de tamanho médio e pequeno.

Palavras-chave: Lycopersicon esculentum Mill., tratos culturais, produção classificada, eficiência fotossintética, rentabilidade.

\section{ABSTRACT}

Tomato plant staking and training systems for fresh fruit production

The main reason for staking and training tomato plants is to keep plants and fruits off the ground to reduce losses and to improve the quality of the production. To evaluate the influence of three plant staking and two training systems of the tomato production for fresh market, two experiments were conducted during the period of August 1999 (exp.1) and 2000 (exp.2) in Viçosa, Brazil. The experimental design was a randomized complete block design with three blocks, in a $3 \times 2$ factorial plant staking and 2 conduction systems. The following staking methods were tested: T1, traditional (two bamboo stakes as an inverted V frame); T2, triangular staking or; T3, vertical staking with polypropylene cord. Plants were conducted with one or two stems per plant. Independent of the treatment, the plants were pruned above the sixth flower cluster. Differences were observed among staking and conduction systems and among the years in relation to the appraised characteristics. There was interaction among training, conduction systems and years for marketable fruits and total fruit production. The vertical staking up provided increase in production of fruits of big size and decrease in production of fruits of medium size and production of not marketable fruits, when compared with the other staking methods. Independently of the conduction system (for two stems), the tomato cultivated with only one stem produced bigger fruits with higher commercial value, while the plants conduced with two stems produced more fruits of medium and small size.

Keywords: Lycopersicon esculentum Mill., cultural treatments, classified production, efficient use of assimilates, profitability.

\section{(Recebido para publicação em 28 de maio de 2004 e aceito em 4 agosto de 2005)}

A cultura do tomateiro é uma das principais hortaliças cultivadas no Brasil. Sua produção anual é de aproximadamente 3,6 milhões de toneladas destinados ao mercado de tomate fresco e para processamento, cultivados em mais de 60 mil ha (AGRIANUAL, 2004). Estão envolvidos, na cultura do tomateiro no Brasil, mais de 10.000 produtores, com aproximadamente 200.000 pessoas trabalhando diretamente na produção desta hortaliça (TAVARES, 2003).

Segundo pesquisa realizada pelo MINISTÉRIO DA INTEGRAÇÃO NACIONAL (2003) o consumidor brasileiro prioriza, na compra dos produ- tos hortícolas, a qualidade e a aparência do produto. No caso do tomate, aspectos como cor, brilho e principalmente o tamanho do fruto têm especial relevância. Tal fato é confirmado pelos preços mais elevados pagos aos frutos de maior tamanho nas Centrais de Abastecimento (CEASAs) espalhadas por todo o Brasil.

Uma das formas de melhoria da qualidade e aparência do tomate produzido é a adoção de técnicas adequadas de manejo da cultura. As principais formas de manejo empregadas na cultura do tomateiro são: tipo de tutoramento, forma de condução e espaçamento. O tipo de tutoramento utilizado, bem como a forma de condução, pode alterar a distribuição da radiação solar e a ventilação em torno das plantas (ANDRIOLO, 1999), influenciando a umidade relativa e a concentração de gás carbônico atmosférico entre e dentro das fileiras (GEISENBERG; STEWART, 1986). O tipo de tratamento também pode influenciar na maior ou menor eficiência de controle de pragas (PICANÇO et al., 1998) e doenças (BOFF et al., 1992).

$\mathrm{O}$ tutoramento mais freqüentemente utilizado pelos agricultores brasileiros é o método tradicional ou "V" invertido (MAKISHIMA; MIRANDA, 1992; FONTES; SILVA, 2002). Nesse 


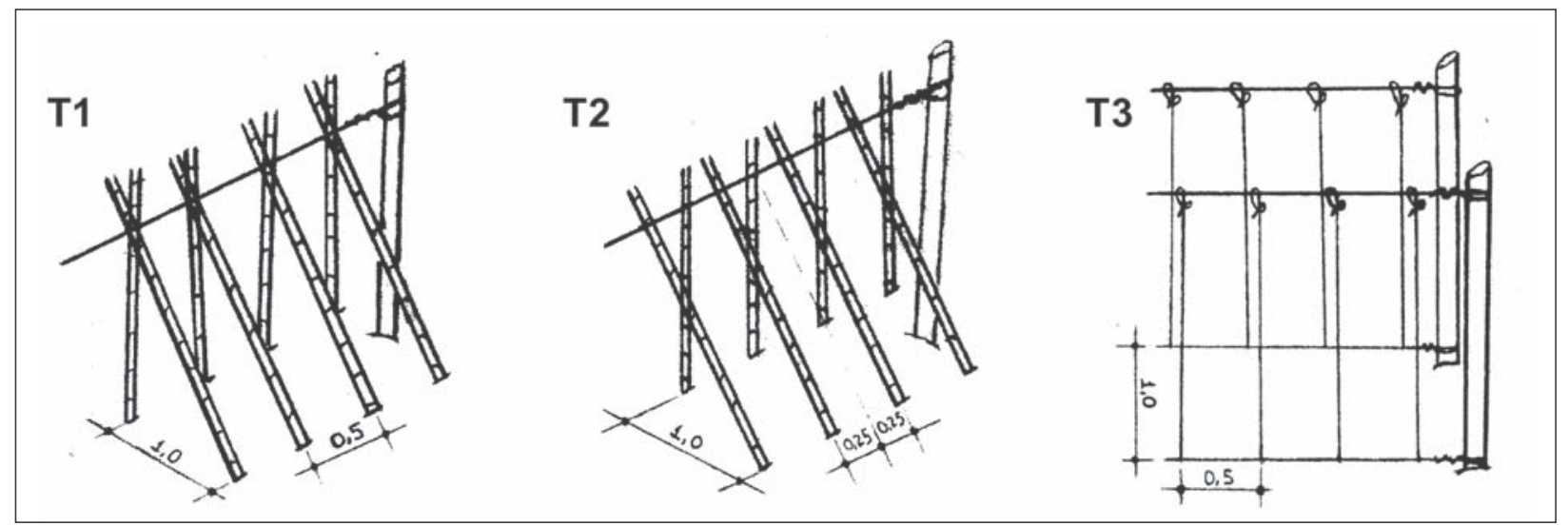

Figura 1. Esquema ilustrativo dos sistemas de tutoramento tradicional ('I'1), triangular ('l'2) e vertical com fitilho ('I'3).

tutoramento ocorre a formação de uma câmara úmida e aquecida sob o "V" invertido, sendo esta, um ambiente favorável ao desenvolvimento de fungos, que ainda ficam livres da ação dos agrotóxicos que são aplicados na parte externa das plantas (REBELO, 1993). Tentando solucionar este problema, propôs-se o tutoramento triangular. Este sistema teoricamente reune as vantagens do método tradicional (estabilidade e não dependência de mão-de-obra especializada) e evita a formação da câmara úmida e aquecida sob o "V" invertido.

Outro método utilizado na cultura do tomateiro é o tutoramento vertical, que permite melhorar a distribuição da radiação solar e a ventilação, reduzindo o período de molhamento foliar e, por conseguinte, a severidade das doenças (ZAMBOLIM et al., 1989).

Além de tutoramento, a planta do tomateiro necessita de condução, podendo, desta forma, maximizar a produção e a qualidade do tomate destinado ao mercado de mesa. Como método de condução considera-se qualquer prática que altere a arquitetura da planta ao longo de seu desenvolvimento, como por exemplo, a poda apical, retirada de brotações laterais, raleio de frutos, entre outros.

A condução da planta com uma haste, sem poda apical ou com poda a 1,80 $\mathrm{m}$ acima do solo, é o método de condução mais utilizado no país (OLIVEIRA et al., 1995; LOPES; STRIPARI, 1998; SILVA et al., 1997). No entanto vários autores têm sugerido a condução das plantas com duas hastes associado à poda apical, devido ao aumento na produtividade proporcionado por estes (FONTES et al., 1987; OLIVEIRA et al., 1995; POERSCHKE et al., 1995;)

A poda apical, deixando-se menor número de cachos por planta, traz benefícios à cultura do tomateiro, tais como: redução do ciclo, facilidade de execução dos tratos culturais, aumento no peso médio dos frutos, redução e maior segurança na aplicação de agrotóxicos (CAMPOS et al., 1987; OLIVEIRA et al., 1995; POERSCHKE et al., 1995). Além disso, a limitação do número de cachos por planta é uma prática recomendada principalmente nos cultivos cujas condições ambientais limitem o crescimento da planta do tomateiro e em consequiência o número de cachos produtivos (OLIVEIRA et al., 1995).

Apesar de existirem vários trabalhos avaliando a influência dos métodos de tutoramento (FONTES et al., 1987; SILVA et al., 1997; SANTOS et al., 1999) e de condução (CAMPOS et al., 1987; OLIVEIRA et al., 1995; 1996; POERSCHKE et al., 1995) na produção do tomateiro, alguns resultados são bastante contraditórios. Além disso, poucos são os trabalhos relacionando estes fatores simultaneamente.

O presente trabalho teve como objetivo avaliar três métodos de tutoramento e dois métodos de condução do tomateiro, podado acima do sexto cacho, quanto à produção classificada de frutos em dois anos consecutivos de cultivo.

\section{MATERIAL E MÉTODOS}

Os experimentos foram realizados em campo, na Horta da Universidade
Federal de Viçosa, de agosto a dezembro de 1999 e 2000, em solo Podzolossolo Vermelho-Amarelo, textura argilosa e topografia plana. A recomendação de adubação foi baseada na $5^{\text {a }}$ Aproximação (Ribeiro et al., 1999).

Os experimentos foram conduzidos em delineamento em blocos ao acaso com três repetições num esquema fatorial $3 \times 2$. Os fatores foram constituídos de três métodos de tutoramento e dois de condução da planta do tomateiro e as parcelas constituídas por quatro fileiras de dez plantas, considerando-se úteis as doze plantas centrais, sendo seis plantas de cada uma das duas fileiras internas da parcela. Foi utilizada a cultivar Santa Clara, tipo Santa Cruz, cujas mudas foram produzidas em bandejas com 128 células preenchidas com substrato comercial.

O espaçamento utilizado foi de $1,0 \times 0,5 \mathrm{~m}$ entre e dentro das fileiras, respectivamente. Na condução dos experimentos foram utilizadas as técnicas recomendadas para o cultivo do tomateiro (MAKISHIMA; MIRANDA, 1992; FILGUEIRA, 2000).

Os métodos de tutoramento (Figura 1) foram:

$\mathrm{T} 1$ - Tutoramento tradicional ou $\mathbf{V}$ invertido ou cerca cruzada (FILGUEIRA, 2000).

T2 - Tutoramento triangular; para se obter este tratamento as fileiras ímpares foram iniciadas imediatamente após os mourões, enquanto as fileiras pares iniciaram a $0,25 \mathrm{~m}$ destes. De forma, que havia distância horizontal de $0,25 \mathrm{~m}$ entre fileiras adjacentes, dando assim a 
conformação de um triângulo entre plantas de fileiras contíguas.

T3 - Tutoramento vertical com fitilho; neste sistema a planta é tutorada por fitilho preso a dois fios de arame horizontais e paralelos, o primeiro a 0,10 $\mathrm{m}$ e o segundo a $1,8 \mathrm{~m}$ em relação ao solo (LOPES; STRIPARI, 1998; FONTES; SILVA, 2002).

Os métodos de condução avaliados foram uma e duas hastes por planta. Independentemente do sistema de tutoramento e do número de hastes por planta; estas foram despontadas mantendo-se seis racimos. O desponte foi efetuado deixando-se três folhas acima do sexto racimo de cada planta.

As colheitas foram feitas em intervalos de sete dias cada, de 30/11 a 29/ $12 / 1999$ e de $21 / 11$ a 27/12/2000, nas quais se avaliaram as seguintes características:

a) produção de frutos de tamanho grande, $\mathrm{PFG}\left(\mathrm{t} \mathrm{ha}^{-1}\right)$; b) produção de frutos de tamanho médio, PFM $\left.\left(\mathrm{t} \mathrm{ha}^{-1}\right) ; \mathrm{c}\right)$ produção de frutos de tamanho pequeno, $\operatorname{PFP}\left(\mathrm{t} \mathrm{ha}^{-1}\right)$; d) produção precoce de frutos de tamanho grande, PPFG $\left(\mathrm{t} \mathrm{ha}^{-1}\right)$, obtida pela soma dos pesos dos frutos desta classe colhidos na primeira e segunda colheita; e) produção precoce de frutos de tamanho médio, PPFM ( $\left.\mathrm{t} \mathrm{ha}^{-1}\right)$, obtida soma dos pesos de frutos desta classe colhidos na primeira e segunda colheita; f) produção de frutos comercializáveis, PC $\left(\mathrm{t} \mathrm{ha}^{-1}\right)$, obtida pela soma dos pesos dos frutos de tamanho grande, médio e pequeno; g) produção de frutos não comercializáveis, PNC (t ha $^{-1}$ ), obtida pela soma dos pesos dos frutos sem classificação comercial; h) produção de frutos com defeitos, PFD $\left(\mathrm{t} \mathrm{ha} \mathrm{a}^{-1}\right.$ ), obtida pela soma dos pesos de todos os frutos com defeitos causados por insetos, pássaros, roedores, doenças, danos mecânicos e fisiológicos e h) produção total, PT ( t ha $\left.^{-1}\right)$, obtida pela soma dos pesos de todos os frutos produzidos. A classificação de frutos de tamanho grande, médio e pequeno seguiu as normas do MINISTÉRIO DA AGRICULTURA, PECUÁRIA E ABASTECIMENTO (2002).

Foram realizadas análises de variância individuais e conjuntas dos experimentos para cada característica (STEEL et al., 1997). Aplicou-se o teste Tukey a

Tabela 1. Análise conjunta ${ }^{1}$ dos dados relativos aos anos de 1999 e 2000 de seis características agronômicas avaliadas em tomateiro cultivado em três sistemas de tutoramento. Viçosa, UFV. 2004.

\begin{tabular}{lllllll}
\hline Tutor $^{2}$ & PFG $^{3}$ & PFM & PFP & PPFG & PNC & PFD \\
\hline T1 & $41,0 \mathrm{ab}$ & $18,7 \mathrm{a}$ & $8,4 \mathrm{a}$ & $7,1 \mathrm{a}$ & $3,6 \mathrm{a}$ & $12,6 \mathrm{a}$ \\
$\mathrm{T} 2$ & $35,8 \mathrm{~b}$ & $20,5 \mathrm{a}$ & $9,1 \mathrm{a}$ & $6,0 \mathrm{a}$ & $4,1 \mathrm{a}$ & $15,2 \mathrm{a}$ \\
T3 & $43,6 \mathrm{a}$ & $17,1 \mathrm{a}$ & $8,2 \mathrm{a}$ & $6,6 \mathrm{a}$ & $2,8 \mathrm{a}$ & $14,4 \mathrm{a}$ \\
\hline C = 2T3- & $10,5^{*}$ & $-4,9^{*}$ & $-1,0^{\mathrm{ns}}$ & $0,1^{\mathrm{ns}}$ & $-2,1^{*}$ & $1,2^{\mathrm{ns}}$ \\
(T1+ T2) & & & &
\end{tabular}

${ }^{1}$ Médias seguidas de pelo menos uma mesma letra, em cada coluna, não diferem entre si, ao nível de $5 \%$ de probabilidade, pelo teste Tukey; ${ }^{*}$,ns Contraste significativo e não significativo, respectivamente, ao nível de $5 \%$ de probabilidade pelo teste de Scheffé; ${ }^{2} \mathrm{~T} 1$ : tutoramento tradicional; T2: tutoramento triangular; T3: tutoramento vertical com fitilho; ${ }^{3} \mathrm{PFG}, \mathrm{PFM} \mathrm{e}$ PFP: produção de frutos de tamanho grande, médio e pequeno, respectivamente; PPFG: produção precoce de frutos de tamanho grande; PNC: produção de frutos não comercializáveis e PFD: produção de frutos com defeitos.

5\% de probabilidade para comparação entre médias. Seguiu-se o método de Scheffé para comparação de contraste. Utilizou-se, para todas as análises, o programa computacional SAEG (RIBEIRO JÚNIOR, 2001).

\section{RESULTADOS E DISCUSSÃO}

Para todas as características, com exceção de PPFM, a relação entre os quadrados médios do resíduo das análises de variâncias individuais dos experimentos, foi inferior a quatro, permitindo que se procedesse à análise conjunta dos experimentos, como citado por Gomes (1970). Para a característica PPFM, utilizou-se o método proposto por Cochran (1954), visando o ajuste dos graus de liberdade, viabilizando a análise conjunta dos experimentos

A partir da análise de variância conjunta dos experimentos verificou-se que houve interação tripla do tipo Tutoramentos*Conduções*Anos significativa para as características PC e PT e interação Tutoramentos*Anos para PPFM. Para as demais características não houve interação, sendo assim, as comparações entre métodos de tutoramento e condução, na análise conjunta dos experimentos, foram realizadas a partir dos valores médios dos dois anos.

Considerando-se apenas os efeitos do fator tutoramento verificou-se, na análise conjunta dos experimentos, que o método de tutoramento vertical foi o mais produtivo para a característica
PFG, não diferindo do método tradicional (Tabela 1). O tutoramento vertical proporcionou aumento de 6,34\% e $21,78 \%$ em relação ao tutoramento tradicional e triangular, respectivamente. Tal resultado tem elevada importância para o produtor, pois os frutos de maior tamanho são aqueles que alcançam maiores preços no mercado para consumo in natura.

Com o intuito de comparar o tutoramento vertical frente aos demais métodos avaliados, realizou-se o contraste $=2 \mathrm{~T} 3-(\mathrm{T} 1+\mathrm{T} 2)$. A partir deste, verificou-se que o sistema vertical proporcionou aumento na produção de frutos de tamanho grande, diminuição na produção de frutos de tamanho médio e de frutos não comerciais (Tabela 1). Além disso, não houve diferença quanto à produção de frutos de tamanho pequeno, produção precoce de frutos de tamanho grande e frutos com defeitos. Estes resultados são concordantes com os encontrados por Santos et al. (1999) que verificaram não haver efeito do método de tutoramento sobre a incidência de doenças no tomateiro e conseqüentemente sobre a produção de frutos com defeitos.

A maior insolação e ventilação propiciada à cultura do tomateiro pelo método vertical, como relatado por Zambolim et al. (1989), resultou, neste trabalho, em maior produção de frutos grandes. Tal fato pode ser explicado, pois maior insolação possibilita maior interceptação da radiação solar fotossinteticamente ativa pela planta e 
Tabela 2. Estimativas ${ }^{1}\left(\right.$ t.ha $\left.^{-1}\right)$ da produção de frutos comercializáveis (PC) e produção total (PT) avaliadas em tomateiro cultivado em três sistemas de tutoramento e duas formas de condução, nos anos de 1999 e 2000. Viçosa, UFV. 2004.

\begin{tabular}{|c|c|c|c|c|c|c|c|c|}
\hline \multirow{3}{*}{ Tutor $^{2}$} & \multicolumn{4}{|c|}{ PC } & \multicolumn{4}{|c|}{ PT } \\
\hline & \multicolumn{2}{|c|}{1999} & \multicolumn{2}{|c|}{2000} & \multicolumn{2}{|c|}{1999} & \multicolumn{2}{|c|}{2000} \\
\hline & 1 haste & 2 hastes & 1 haste & 2 hastes & 1 haste & 2 hastes & 1 haste & 2 hastes \\
\hline T1 & 73,4 a $A$ & 61,1 a $B$ & 70,4 a $A$ & 67,3 a $A$ & 90,5 a $A$ & 78,3 a $B$ & 86,8 a $A$ & 81,4 a $A$ \\
\hline T2 & 59,9 b B & 69,4 a $A$ & 71,0 a $A$ & 60,9 a $A$ & 80,9 a $A$ & 91,2 a $A$ & 86,9 a $A$ & 79,4 a $A$ \\
\hline T3 & $69,3 \mathrm{ab} A$ & 65,0 a $A$ & 71,3 a $A$ & 70,2 a $A$ & 92,5 a $A$ & 81,3 a $B$ & 86,1 a $A$ & 84,9 a $A$ \\
\hline
\end{tabular}

${ }^{1}$ Médias seguidas de pelo menos uma mesma letra minúscula, na vertical, e maiúsculas, na horizontal, não diferem entre si, ao nível de 5\% de probabilidade, pelo teste Tukey; ${ }^{2} \mathrm{~T} 1$ : tutoramento tradicional; T2: tutoramento triangular; T3: tutoramento vertical com fitilho.

Tabela 3. Análise conjunta dos dados relativos a sete ${ }^{1}$ características agronômicas avaliadas em tomateiro cultivado em duas formas de condução nos anos de 1999 e 2000. Viçosa, UFV. 2004.

\begin{tabular}{lccccccc}
\hline $\mathbf{N}^{\circ}$ hastes & PFG* $^{*}$ & PFM $^{*}$ & PFP* $^{*}$ & PPFG* $^{*}$ & PPFMns & PNCns & PFDns \\
\hline 1 & 45,8 & 16,4 & 7,0 & 8,0 & 4,0 & 3,0 & 15,0 \\
2 & 34,4 & 21,2 & 10,1 & 5,2 & 4,6 & 4,0 & 13,1 \\
\hline
\end{tabular}

*,ns Significativo e não significativo, respectivamente, ao nível de 5\% de probabilidade pelo teste F; ${ }^{1} \mathrm{PFG}$, PFM e PFP: produção de frutos de tamanho grande, médio e pequeno, respectivamente; PPFG e PPFM: produção precoce de frutos de tamanho grande e médio, respectivamente; PNC: produção de frutos não comercializáveis e PFD: produção de frutos com defeitos.

maior ventilação reduz a umidade relativa do ar e renova a concentração de gás carbônico na atmosfera adjacente às folhas, resultando, desta forma, em maior eficiência fotossintética (LOOMIS; AMTHOR, 1999). Esta hipótese é reforçada observando-se a característica PNC (Tabela 1), na qual o tutoramento vertical possibilitou diminuição na sua produção, ou seja, possivelmente uma maior eficiência fotossintética pode ter proporcionado maior disponibilização de fotoassimilados que, direcionados aos frutos, aumentaram o tamanho destes.

Para as características PC e PT foram realizadas as análises individuais dos experimentos, sendo estas avaliadas dentro de cada nível do fator condução (Tabela 2). Verificou-se diferença entre os métodos de tutoramento apenas para a característica PC, no ano de 1999 e em plantas conduzidas com uma haste. Neste caso, as maiores médias foram observadas nas parcelas com os tutoramentos tradicional e vertical (Tabela 2).

Em relação aos métodos de condução (Tabela 3), observou-se que plantas conduzidas com uma haste produziram mais frutos de tamanho grande e com maior precocidade. Já as plantas conduzidas com duas hastes foram mais produtivas para as características PFM e PFP. Não houve diferença entre os dois métodos de condução quanto às características PPFM, PNC e PFD.

Para a característica PC no ano de 1999 e nas parcelas com o tutoramento tradicional, plantas conduzidas com uma haste produziram mais, quando comparadas com aquelas conduzidas com duas hastes. Já naquelas com o método triangular, observou-se maiores médias para plantas conduzidas com duas hastes (Tabela 2). Para PT, plantas conduzidas com uma haste foram mais produtivas nas parcelas tutoradas com os métodos tradicional e vertical (Tabela 3) e não diferiram daquelas conduzidas com duas hastes nas parcelas com o método triangular. Em 2000, não houve efeito de condução em nenhum nível do fator tutoramento.

Estes resultados discordam dos encontrados por Oliveira et al. (1995) que em trabalho realizado em condições (período, cultivar, local) bastante semelhantes ao deste, verificaram que não houve efeito para produção de frutos grandes (diâmetro maior que $60 \mathrm{~mm}$ ). Além disso, verificaram efeito significativo para PC e PT, com menor produção para plantas conduzidas com uma haste. Isso pode ter ocorrido, pois Oliveira et al. (1995) utilizaram espaçamento de $1,0 \times 0,6 \mathrm{~m}$, enquanto neste trabalho o espaçamento adotado foi de 1,0x0,5 m. Possivelmente, no espaçamento $0,5 \mathrm{~m}$ entre plantas a condução das plantas com duas hastes tenha resultado em maior competição por fotoassimilados, com conseqüente redução no peso médio dos frutos. No tomateiro, o aumento da densidade de plantas resulta em ganhos quantitativos e, principalmente, qualitativos na produção quando associada com práticas de condução que reduzam a competição por radiação (BORRAZ et al., 1991; STRECK et al., 1998).

Diante dos resultados do presente trabalho, conclui-se que o método de tutoramento triangular não proporcionou vantagens produtivas para a cultura do tomateiro. Enquanto isso, o tutoramento vertical, dentre os avaliados, é o mais adequado para a produção de frutos para o consumo in natura.

Com relação à forma de condução, o cultivo com uma haste, independentemente do método de tutoramento utilizado, proporcionou aumento na produção de frutos grandes. Desta forma, pode-se unir as duas recomendações, ou seja, utilizar, no espaçamento $1,0 \mathrm{x} 0,5 \mathrm{~m}$ e plantas conduzidas com seis cachos, o tutoramento vertical com uma haste, pois, estes proporcionam aumento na produção de frutos grandes sem alteração na produção comercial e total, promovendo assim aumento na receita desta cultura. 


\section{LITERATURA CITADA}

ANDRIOLO, J.L. Fisiologia das culturas protegidas. 1. ed. Santa Maria: UFSM, 1999. 142 p. ANUÁRIO DA AGRICULTURA BRASILEIRA - AGRIANUAL 2004. Campo Grande: FNP Consultoria e Comércio, 2004. 494 p. BOFF, P.; FONTES, P.C.R.; VALE, F.X.; ZAMBOLIM, L. Controle da Mancha-deEstenfílio e da Pinta-Preta do tomateiro em função do sistema de condução. Horticultura Brasileira, Brasília, v.10, n.1, p.25-27. 1992.

BORRAZ, J.C.; CASTILHO, F.S.; ROBLES, P.E Efectos del despunte y la densidade de poblacion sobre variedades de tomate (Licopersicum esculentum Mill), em hidroponia bajo invernadero. Revista Chapingo, v.15, n.73/74, p.26-30, 1991. CAMPOS, J.P.; BELFORD, C.C.; GALVÃO, J.D.; FONTES, P.C.R. Efeito da poda da haste e da população de plantas sobre a produção do tomateiro. Revista Ceres, Viçosa, v.34, n.192, p.198208, 1987.

COCHRAN, W.G. The combination of estimates from different experiments. Biometrics. n.10, p.101-129. 1954

FILGUEIRA, F.A.R. Novo manual de Olericultura: Agrotecnologia moderna na produ ção e comercialização de hortaliças. 2. ed. Viçosa: Editora UFV, 2000. 402 p.

FONTES, P.C.R.; NAZAR, R.A.; CAMPOS, J.P Produção e rentabilidade da cultura do tomateiro, afetadas pela fertilização e pelo sistema de condução. Revista Ceres. Viçosa, v.34, n.134, p.35565, 1987.

FONTES, P.C.R.; SILVA, D.J.H. Produção de tomate de mesa. 1. ed. Viçosa: Aprenda Fácil, 2002. 193 p.

GEISENBERG, C.; STEWART, K. Field crop management. In: ATHERTON, J.G.; RUDICH, J. (Ed.) The tomato crop. London: Chapman \& Hall, 1986. cap.13, p.511-557.

GOMES, F.P. Curso de estatística experimental. 4. ed., São Paulo: Livraria Nobel, 1970. 464 p.
LOOMIS, R.S.; AMTHOR, J.S. Yield potential, plant assimilatory capacity, and metabolic efficiencies. Crop Science, v.39, p.1584-1596, 1999.

LOPES, M.C.; STRIPARI, P.C. A cultura do tomateiro. In: GOTO, R.; TIVELLI, S.W. Produção de hortaliças em ambiente protegido: condições subtropicais. São Paulo: Fundação Editora da UNESP, 1998. cap.9, p.257-319.

MAKISHIMA, N.; MIRANDA, J.E.C. (Ed.) Cultivo do Tomate (Lycopersicon esculentum Mill.) -. Brasília: CNPH, 1992. 22 p. (Instruções Técnicas do CNPH N. $\left.{ }^{\circ} 11\right)$.

MINISTÉRIO DA AGRICULTURA PECUÁRIA EABASTECIMENTO - MAPA. Normas de identificação, qualidade, acondicionamento, embalagem e apresentação do tomate - Portaria $\mathrm{N}^{\circ} 85$, publicada no Diário Oficial da União de 18/03/ 2002.

MINISTÉRIO DA INTEGRAÇÃO NACIONAL - MIN. Pesquisa em Minas e Rio revela o consumidor de hortifrúti. Frutifatos. Out 2003, n.4, p.1230, 2003.

OLIVEIRA, V.R.; CAMPOS, J.P.; FONTES, P.C.R.; REIS, F.P. Efeito do número de hastes por planta e poda apical na produção classificada de frutos de tomateiro. Ciência e Prática, Lavras, v.19, n.4, p.414-419, 1995.

OLIVEIRA, V.R.; FONTES, P.C.R.; CAMPOS, J.P.; REIS, F.P. Qualidade do tomate afetada pelo número de ramos por planta e pela poda apical. Revista Ceres, Viçosa, v.43, n.247, p.309-318, 1996.

PICANÇ, M.C.; LEITE G.L.D.; GUEDES, R.N.C.; SILVA, E.E.A. Effect of spacing and chemical control on losses and pest attacks on tomato plant with vertical tutoring in Brazil. Crop Protection, v.17, p.447-452, 1998.

POERSCHKE, P.R.C.; BURIOL, G.A.; STRECK N.A.; ESTEFANEL, V. Efeito de sistemas de poda sobre o rendimento do tomateiro cultivado em estufa de polietileno. Ciência Rural, Santa Maria, v. 25, n.3, p.379-384, 1995
REBELO, J.A. Sistema alternativo de tutoramento para tomateiro. Horticultura Brasileira, Brasília, v.11, n.2, p.161, 1993.

RIBEIRO JÚNIOR, J.I. Análises estatísticas no SAEG. Viçosa: Editora UFV, 2001. 301 p.

RIBEIRO, A.C.; GUIMARÃES, P.T.G.; ALVAREZ, V.; V.H. (Ed.) Recomendações para o uso de corretivos e fertilizantes em Minas Ge rais $-5^{a}$ Aproximação. Viçosa: CFSEMG, 1999. $359 \mathrm{p}$.

SANTOS, H.S.; PERIN, W.H.; TITATO, L.G. VIDA, J.B.; CALLEGARI, O. Avaliação de sistemas de condução em relação à severidade de doenças e à produção de tomateiro. Acta Scientiarum, v.21, n.3, p.453-457. 1999.

SILVA, D.J.H.; SEDIYAMA, M.A.N.; MATA, A.C.; ROCHA, D.M.; PICANÇO, M.C. Produção de frutos de tomateiro (Lycopersicon esculentum Mill) em quatro sistemas de cultivo. Revista Ceres, Brasília, v.44, n.252, p.129-141, 1997.

STEEL, R.G.D.; TORRIE, J.H.; DICKEY, D.A. Principles and procedures of statistics a biometrical approach. 3. ed. New York: McGraw - Hill, 1997. 666 p

STRECK, N.A; BURIOL, G.A; ANDRIOLO, J.L.; SANDRI, M.A. Influência da densidade de plantas e da poda apical drástica na produtividade do tomateiro em estufa de plástico. Pesquisa Agropecuária Brasileira, Brasília, v.33, n.7, p.1105-1112, 1998

TAVARES, C.A.M. Ataque dos vírus. Cultivar Hortaliças e Frutas. Dez2002/Jan 2003, p. 26-29, 2003.

ZAMBOLIM, L.; VALE, F.X.R.; CRUZ FILHO, J.; CHAVES, G.M. Controle integrado das doenças do tomateiro. In: ENCONTRO NACIONAL DE PRODUÇÃO E ABASTECIMENTO DE TOMATE, 1, 1989, Viçosa. Anais... Viçosa: UFV, 1989. p. $55-76$ 